\title{
Impact of different antimycotics on cytokine levels in an in vitro aspergillosis model in human whole blood
}

\author{
Zoe Oesterreicher $^{1} \cdot$ Sabine Eberl ${ }^{1} \cdot$ Markus Zeitlinger $^{1}$
}

Received: 29 April 2019 / Accepted: 25 July 2019 / Published online: 1 August 2019

(c) The Author(s) 2019

\begin{abstract}
Background Although fungal infections play a central role in severely ill and immunosuppressed patients, in contrast to antibiotics immunomodulatory effects of antifungals have not been sufficiently investigated. The present study sets out to compare the effect of different antimycotics on immunologic reaction towards mold in vitro.

Materials/methods Aspergillus fumigatus ATCC204305 was used to develop a model of invasive aspergillosis in vitro in whole blood. Since autoclaved hyphal suspension demonstrated the most potent cytokine release, they were used for the further study including blood of 20 male volunteers. Impact on IL-6, IL-8 and TNF- $\alpha$ time concentration profiles by $5 \mathrm{mg} /$ $\mathrm{mL}$ conventional and liposomal amphotericin $\mathrm{B}, 20 \mathrm{mg} / \mathrm{mL}$ fluconazole, $5 \mathrm{mg} / \mathrm{mL}$ voriconazole, $2 \mathrm{mg} / \mathrm{mL}$ posaconazole or saline solution was investigated over $4 \mathrm{~h}$ of incubation at $37^{\circ} \mathrm{C}$.

Results Compared to baseline, cytokine levels increased by addition of hyphal suspension over $4 \mathrm{~h}$ approximately: 54-fold for IL-6, 1000-fold for IL-8 and 270-fold for TNF- $\alpha$. While conventional amphotericin B further increased IL-6 and to a smaller extent IL-8 levels, this was not the case for its liposomal formulation. Congruently amphotericin B increased cytokines in blood without fungus substantially. Fluconazole reduced cytokine increase for all three cytokines compared to stimulation with hyphae without antifungal agent.

Conclusions Our data indicate significant differences in the immunomodulatory potency of different antimycotics. While fluconazole had the highest anti-inflammatory potential, conventional amphotericin even increased cytokine release. This preliminary information might have clinical implication, since cytokine dysregulation plays a major role in the pathogenesis and outcome of fungal infections. Clinical studies are warranted to confirm our findings.
\end{abstract}

Keywords Immunomodulatory effect $\cdot$ Antimycotics $\cdot$ Cytokines $\cdot$ Aspergillosis

\section{Introduction}

The inflammatory response during sepsis has been recognized as cause for progressive systemic organ failure. TNF- $\alpha$ was demonstrated to induce a progressive decrease in cardiovascular function, [1] and high level of IL-6 correlate with a significant mortality increase in septic patients [2]. However, several clinical studies have failed to show a sustained survival benefit from reducing immune reaction by administering anti-endotoxin antibodies, anti-TNF antibodies or soluble TNF- $\alpha$ receptors $[3,4]$. For antibiotics, previous studies have pointed out potential immunomodulatory

Markus Zeitlinger

markus.zeitlinger@meduniwien.ac.at

1 Department of Clinical Pharmacology, Medical University of Vienna, Waehringer Guertel 18-20, 1090 Vienna, Austria effects apart from their antimicrobial effect. This has been shown among others for macrolides [5-7], tetracyclines [7, 8], and fluoroquinolone. [7, 9, 10] Apart from data on the immunomodulatory effect in vitro and in vivo, studies on the clinical implication of the immunomodulatory characteristics of clarithromycin administration showed a considerable benefit in time to resolution of ventilator-associated pneumonia compared to placebo as well as improvement of mortality [3,11]. Even when sepsis was caused by pneumonia due to macrolide-resistant pathogens, improved mortality rates point out that non-antimicrobial effects are the underlying cause of benefit [12].

Besides and on top of bacterial sepsis, invasive fungal infections are increasingly problematic for severely ill and immunosuppressed patients, especially for those receiving intensive cytostatic treatment for acute leukemia, hematopoietic stem cell transplants (HSCT) or solid organ transplants, 
causing considerable morbidity and mortality rates [13]. In particular numbers of invasive fungal infections caused by molds such as Aspergillus are increasing [14] and invasive pulmonary aspergillosis is the most common cause. Even when sepsis was caused be for pneumonia-related mortality in patients with leukemia or HSCT recipients showing an overall mortality rate of invasive aspergillosis of $58-87 \%$ in patients who receive bone marrow transplants [15].

Cytokine dysregulation seems to play a major role in the pathogenesis and outcome of fungal infections [16, 17]. Potential pathways of dysregulation may include binding of fungal ligands and pattern recognition receptors, like Tolllike receptors, on innate immune cells. Thereby intracellular signaling cascades are activated and lead to an inflammatory response in the host, beneficial for the patient or not [17]. Antifungals play an essential role in current treatment and prophylaxis in this population $[16,18,19]$ and more information about potential immunomodulatory effect of antifungal agents in analogy to antibacterial agents would be desirable.

The present study aimed to establish an in vitro aspergillosis model in whole blood to study the immunomodulatory effect of antimycotics.

\section{Methods}

\section{General information and ethics}

This study was performed at the Department of Clinical Pharmacology, Medical University of Vienna, Austria. It was conducted in accordance with the Declaration of Helsinki and the current revisions of the Good Clinical Practice Guidelines of the European Commission and the Good Scientific Practice Guidelines of the Medical University of Vienna. The protocol was approved by the Ethics Committee of the Medical University of Vienna (EK Number 1045/2015). Informed consent was sought from all healthy volunteers prior to blood collection.

\section{Study subjects}

27 healthy male volunteers were included in this trial. Inclusion criteria were age between 19 and 40 years as well as laboratory parameters within the given range or abnormal parameters which were not clinically relevant. None of the volunteers had concomitant medication within 1 week or symptoms of clinically relevant illness within 3 weeks prior to the study day. Furthermore, subjects were excluded if they were smoking more than 5 cigarettes per day or had treatment with an investigational drug within 3 weeks or blood donation within 4 weeks before the study.

\section{Blood sampling}

During the pilot of the study blood samples were collected by venipuncture using Vacutainer tubes anticoagulated with EDTA (Vacuette ${ }^{\circledR}$ ). The first experiments of adding both hyphal fragments and antimycotic agents showed that blood samples became hemolytic using EDTA tubes. To optimize the model and avoid hemolysis, Citrate tubes were used for blood sampling and Falcon Tubes (TPP Techno Plastic Products AG) for incubation in the main part.

\section{Establishment of aspergillosis model}

For establishment of an aspergillosis model in human whole blood in vitro blood samples from the first 7 volunteers was used. The cytokine time profiles induced by different conidia counts $\left(10^{7}\right.$ conidia/mL and $10^{8}$ conidia/ $\mathrm{mL}$ ) and inactivated as well as active hyphal fragments at different concentrations obtained from a prior conidia count of $10^{6}$ conidia/mL were compared with cytokine time profiles induced by LPS (US Standard Reference Endotoxin E. coli, CC-RE-Lot 2) $50 \mathrm{pg} / \mathrm{mL}$ and negative (saline) control. This comparison was performed to identify the stimulus - conidia or hyphal fragments - of greatest potency on cytokine release. LPS concentration of $50 \mathrm{pg} / \mathrm{mL}$ was used congruently with a prior study [20]. The cultivation of conidia and hyphae was performed as follows:

Cultivation of conidia Aspergillus fumigatus ATCC204305 was cultured for 2-4 days on Sabouraud Dextrose agar (Becton-Dickinson, Netherlands). After the incubation time, $3-5 \mathrm{~mL}$ of spore buffer was pipetted on the Sabouraud agar plate and conidia were scraped from the plate using an inoculation loop. The suspension of spore buffer containing conidia was collected with a single use pipette and filtered with a cell retainer $(70 \mu \mathrm{m})$. Conidia were diluted 100-1000-fold and counted in a hemocytometer (Bürker Türk Kammer, VWR Merck). Conidia suspensions containing different counts per $\mathrm{mL}$ $\left(10^{7}\right.$ conidia $/ \mathrm{mL}$ and $10^{8}$ conidia $\left./ \mathrm{mL}\right)$ were stored at $-4{ }^{\circ} \mathrm{C}$.

Cultivation of hyphae $30 \mathrm{~mL}$ of the conidia suspension containing $10^{6}$ conidia/mL was inoculated into $30 \mathrm{~mL}$ of Nitrogen Base in Erlenmeyer flasks. Incubation was performed for approximately $16 \mathrm{~h}$ at $37^{\circ}$ at a shaker before the content of the Erlenmeyer flasks was centrifuged at $4000 \mathrm{rpm}$ for $15 \mathrm{~min}$. The pellets were washed twice in HBSS and then resuspended in PBS (Lonza, Basel, Swiss) to achieve the same volume as initially added as conidia suspension. For inactivating hyphal fragments, the gained 
pellets were then autoclaved at $121{ }^{\circ} \mathrm{C}$ for $15 \mathrm{~min}$ in a glass tube. As inactivation control, a sample was placed on one Sabouraud Dextrose agar plate (Becton-Dickinson, Netherlands) and observed for 3 days for lack of growth after incubation. The further procedure was the same for both, inactivated and vital hyphal fragments. The pellets were washed twice with HBSS (Gibco/Life Technologies) and finally again resuspended in HBSS in the same volume as initially added as conidia suspension. For all experiments with hyphae either $1 \mathrm{~mL}$ of solution of vital or of inactivated hyphae was added to human whole blood for further incubation.

Establishment of the aspergillosis model Aspergillus fumigatus conidia suspension of different conidia counts of $10^{7} / \mathrm{mL}$ and $10^{8} / \mathrm{mL}$, as well as active and inactivated hyphal suspensions containing hyphae starting from the prior conidia count of $10^{6}$ conidia/mL were studied. To $4 \mathrm{~mL}$ of whole human blood in 14-mL falcon tubes, either $1 \mathrm{~mL}$ of the hyphal suspension, or conidia suspension getting a low and a high conidia concentration of finally $7 \times 10^{5}$ or $7 \times 10^{6}$ conidia/mL, or LPS getting a final concentration of $50 \mathrm{pg} / \mathrm{mL}$ or saline solution as negative control were added, respectively. Before incubation, baseline samples were taken without any addition of stimuli. All blood samples were then incubated at $37^{\circ}$ and samples were taken at $2 \mathrm{~h}, 4 \mathrm{~h}$ or $6 \mathrm{~h}$, $8 \mathrm{~h}$ and $24 \mathrm{~h}$ after start of incubation. Samples were centrifuged at $2500 \mathrm{~g}$ for $10 \mathrm{~min}$ and plasma pipetted into Cryo Vials for storage at $-80^{\circ} \mathrm{C}$ before ELISA was performed. The levels of IL- 6 and TNF- $\alpha$ were determined.

Several inocula (conidia and hyphal fragments) were tested as described above, but solely one was used for the final aspergillosis model in the main part of the study. Thereby the impact of several antifungal agents with and without addition of $A$. fumigatus could be tested for each individual with a justifiable amount of blood. Since inactivated hyphal fragments starting from a conidia count of $10^{6}$ conidia $/ \mathrm{mL}$ demonstrated the most potent cytokine release when compared to conidia or active hyphal fragments, this inoculum was used. Duration of the main experiments was limited to $4 \mathrm{~h}$ since, in line with previous experience from LPS experiments maximum cytokine release was found for this time-point for the majority of subjects.

\section{Main part: incubation of antimycotics in the established model}

20 participants were included in the main part of the study on the impact of antimycotics on cytokine levels in the established aspergillosis model with hyphal fragments. $1 \mathrm{~mL}$ of hyphal suspension obtained from an original suspension containing $10^{6}$ conidia $/ \mathrm{mL}$, or saline solution were added to $4 \mathrm{~mL}$ of whole blood. All experiments were performed as duplicates. The impact of final concentrations of
$5 \mathrm{mg} / \mathrm{L}$ conventional and $5 \mathrm{mg} / \mathrm{L}$ liposomal amphotericin B, $20 \mathrm{mg} / \mathrm{L}$ fluconazole, $5 \mathrm{mg} / \mathrm{L}$ voriconazole and $2 \mathrm{mg} / \mathrm{L}$ posaconazole or saline control was investigated. As negative control, the cytokine levels in whole blood without any stimuli (neither hyphal fragments nor antimycotics) was used, while the control with Aspergillus but no antimycotics is referred to as 'aspergillosis control'. All samples were incubated at $37^{\circ} \mathrm{C}$ over $4 \mathrm{~h}$ at a slow rotation rate imitating physiologic conditions.

Concentrations for antimycotics used were chosen at or marginally above the clinically achieved maximal serum concentration which were $1.7 \mathrm{mg} / \mathrm{L}$ for liposomal amphotericin B when administering $5.0 \mathrm{mg} / \mathrm{kg} /$ day [21], $18 \mathrm{mg} / \mathrm{L}$ for fluconazole at steady state after administration of $400 \mathrm{mg}$ [22], 2-6 $\mu \mathrm{g} / \mathrm{L}$ for voriconazole [23] and $1.3 \mu \mathrm{g} / \mathrm{mL}$ for posaconazole [24]. Concentration of conventional amphotericin $\mathrm{B}$ was chosen to be identical with liposomal amphotericin B to allow comparison of the gained data. Fluconazole was included in this study though it is lacking inhibiting potency against $A$. fumigatus for comparison reason as well as the fact that the anti-inflammatory potency of substances might be attractive independent from any concrete infection.

\section{ELISA for IL-6, IL-8 and TNF-a}

In this study levels of cytokines, IL-6, TNF- $\alpha$ and IL- 8 were determined in plasma using commercially available Elisa kits (Quantikine ${ }^{\circledR}, \mathrm{R} \& \mathrm{D}$ Systems, Austria) according to the manufacturer's instructions. The minimal detection limits were $0.7 \mathrm{ng} / \mathrm{mL}$ for IL-6, between 1.5 and $7.5 \mathrm{pg} / \mathrm{mL}$ for IL- 8 and between 0.5 and $5.5 \mathrm{pg} / \mathrm{mL}$ for TNF- $\alpha$. The minimal detectable dose for this Elisa kits had been previously determined by adding two standard deviations to the mean optical density value of 20 zero standard replicates and calculating the corresponding concentration. In this study, IL-6, IL-8 and TNF- $\alpha$ levels were quantified for all samples at the predefined time points. The results are expressed in $\mathrm{pg} / \mathrm{mL}$.

\section{Analysis and figures}

Data in the text are expressed as means \pm standard deviation of experiments performed in duplicates. In the pilot, 7 subjects were included. In the main part, the whole blood from 10 subjects was assigned to experiments to determine the impact of amphotericin B and fluconazole, while the other 10 subjects were assigned to liposomal amphotericin B, posaconazole and voriconazole. Negative controls with saline solution as well as aspergillosis control adding solely hyphae but no antimycotic agents were performed for all 20 subjects in the main part. Time interleukin concentration profiles were performed using the means of 7 (pilot), 10 (main part) or 20 (main part controls) subjects and are depicted in Figs. 1 and 2. Mean cytokine concentrations 

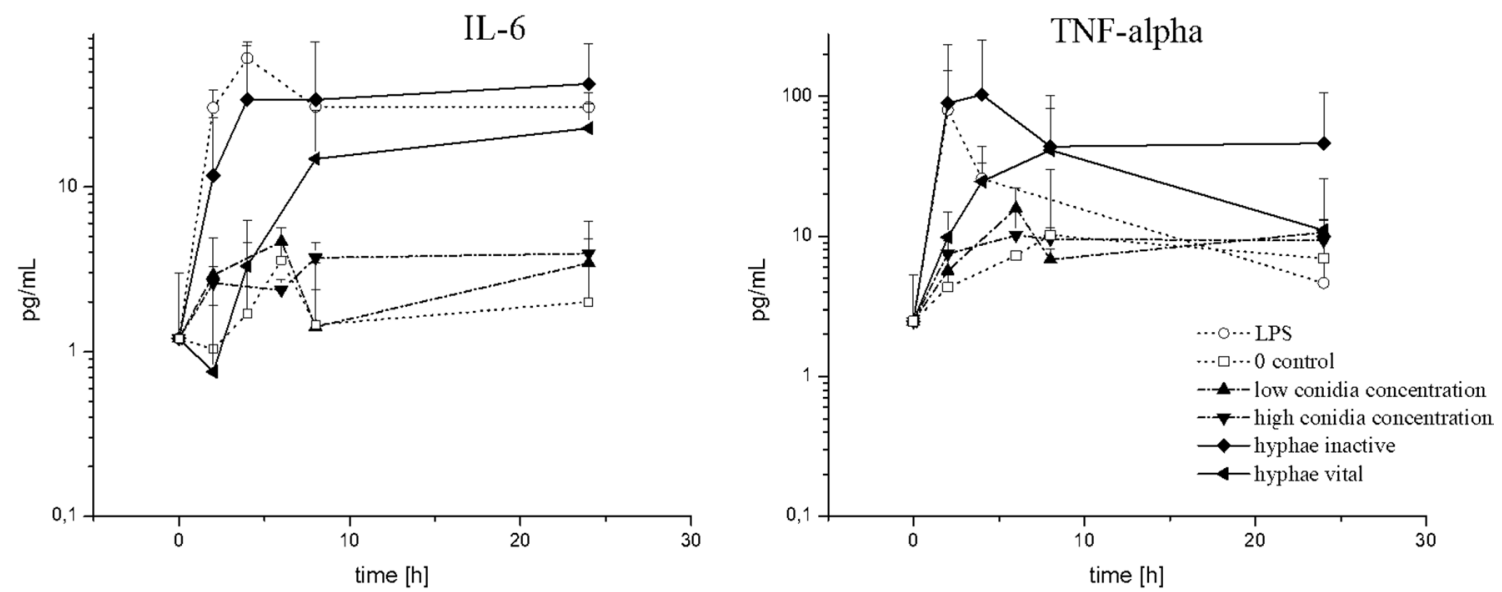

Fig. 1 Mean $( \pm$ SD) concentration vs. time profile of IL-6, and TNF- $\alpha$ in whole blood after stimulation with different concentrations of conidia (low and high conidia count), vital as well as inactivated hyphal fragments of A. fumigatus, LPS and control over $24 \mathrm{~h}$
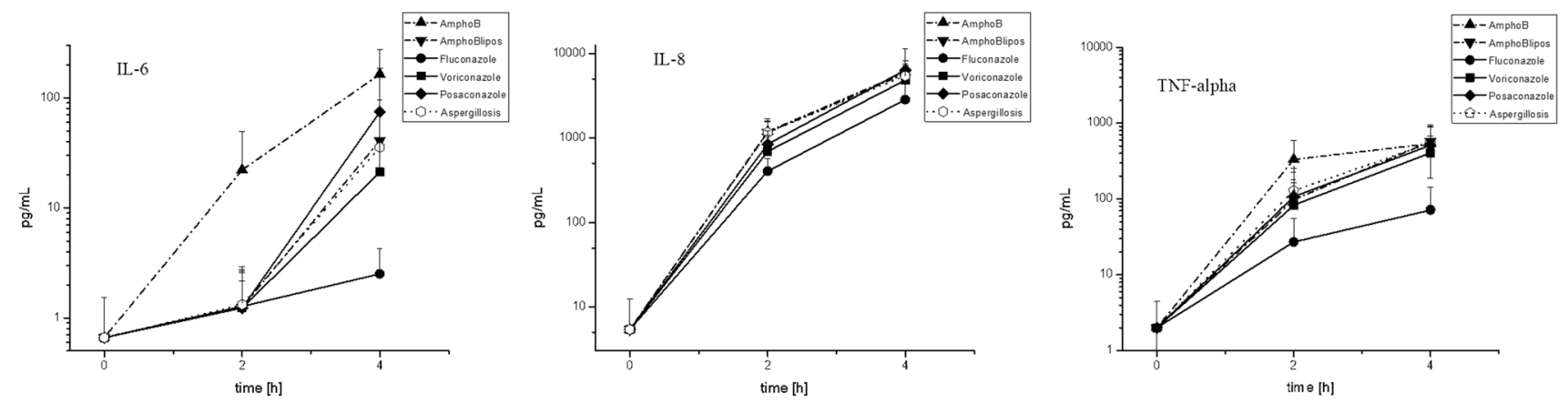

Fig. 2 Mean $( \pm \mathrm{SD})$ concentration vs. time profile of IL-6, IL-8 and TNF- $\alpha$ in whole blood after stimulation with hyphal fragments of $A$. fumigatus with or without addition of different antifungal agents. Aspergillosis control is depicted in open symbols

after $4 \mathrm{~h}$ of incubation are compared in Fig. 3. Ratios were calculated by dividing the quantified cytokine concentration in the presence of an antimycotic by the quantified cytokine concentration in the Aspergillus control sample without antimycotic (aspergillosis control). As well as the ratios of cytokine concentrations after incubation without mold were calculated comparing cytokine values after incubation with antimycotics over zero control (cytokine levels after incubation of whole blood alone).

\section{Results}

\section{Immunomodulatory effect of conidia and hyphal fragments}

For the establishment of an aspergillosis model in human whole blood, the impact of high and low concentration of conidia as well as active or inactivated hyphae of Aspergillus fumigatus hyphae on IL- 6 and TNF- $\alpha$ profiles was determined. Cytokine level profiles over $24 \mathrm{~h}$ are shown in Fig. 1.

Regarding the maximal cytokine levels, for both cytokines, inactivated hyphal fragments showed the greatest cytokine levels among all fungal stimuli and a substantially greater cytokine release than vital hyphae. In contrast, conidia had the smallest impact on cytokine release, with no relevant difference between the two conidia concentrations used. Maximal levels $\left(C_{\max }\right)$ were for both cytokine observed after $4 \mathrm{~h}$. LPS led to an IL-6 increase and TNF- $\alpha$ increase comparable to inactivated hyphal fragments. In absolute numbers, the maximal reached IL- 6 and TNF- $\alpha$ levels were $42.32 \pm 31.56 \mathrm{pg} /$ $\mathrm{mL}$ and $102.90 \pm 148.57 \mathrm{pg} / \mathrm{mL}$ for inactive hyphal fragments, $4.65 \pm 1.03 \mathrm{pg} / \mathrm{mL}$ and $15.80 \pm 6.31 \mathrm{pg} / \mathrm{mL}$ for conidia, and $60.48 \pm 1.32 \mathrm{pg} / \mathrm{mL}$ and $79.87 \pm 71.84 \mathrm{pg} /$ $\mathrm{mL}$ for LPS. While inactive hyphae thereby led up to a cytokine increase of 43-fold for IL-6 and 69-fold for TNF$\alpha$, conidia solely increased IL- 6 and TNF- $\alpha$ levels 2 - and 4-fold, respectively. 

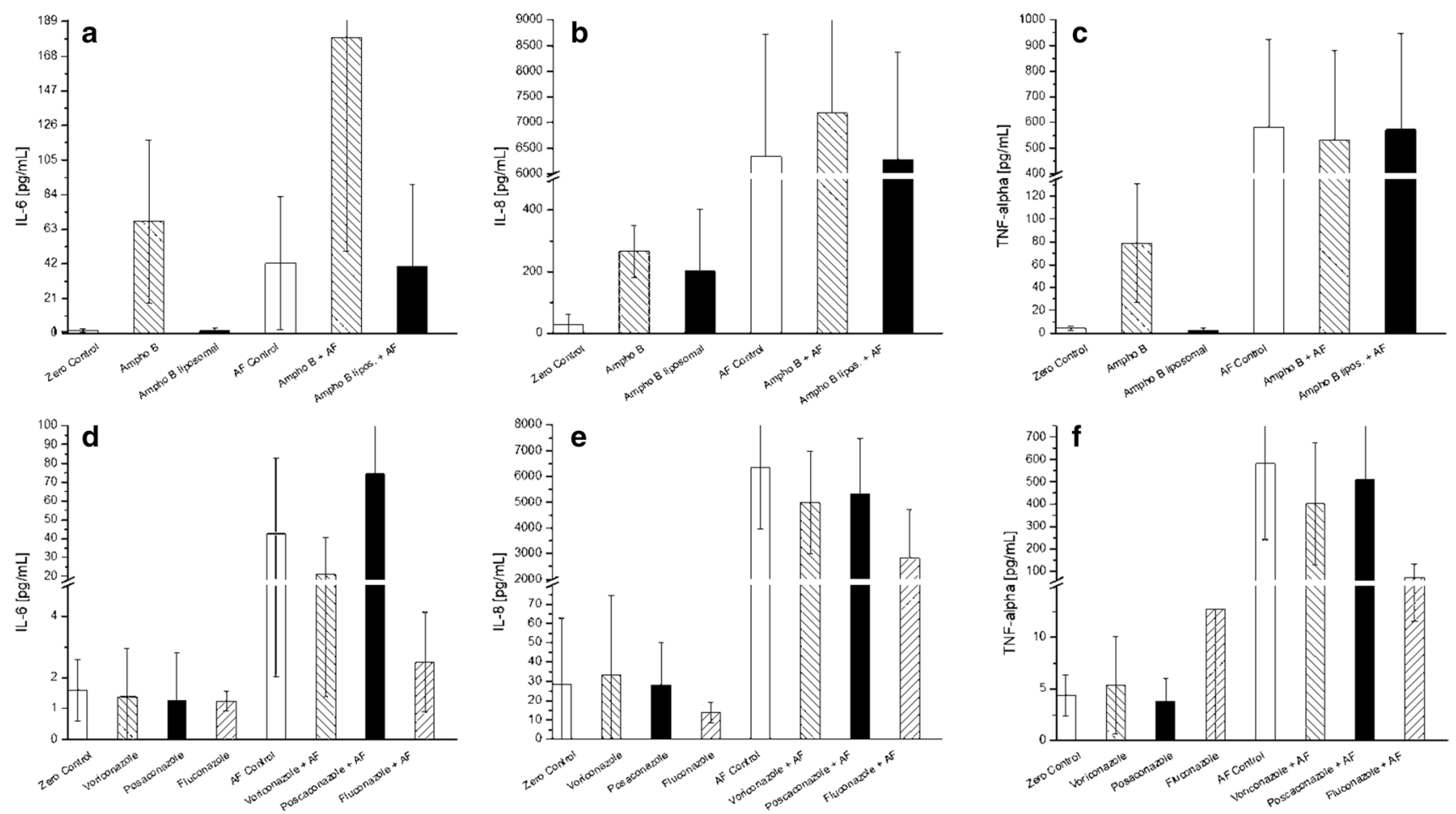

Fig. 3 a-f Absolute cytokine concentrations of IL-6, IL-8 and TNF- $\alpha$ (from left to right) as mean values $( \pm \mathrm{SD})$ after $4 \mathrm{~h}$ of incubation. a-c The impact of conventional amphotericin B and liposomal amphotericin B; d-f the impact of three azole drugs. Each figure depicts

\section{Immunomodulatory effect of antimycotics}

IL-6, IL- 8 and TNF- $\alpha$ profiles were determined at baseline, $2 \mathrm{~h}$ and $4 \mathrm{~h}$ after incubation in the aspergillosis model using inactivated hyphal fragments. Compared to baseline cytokine levels increased by addition of hyphal suspension over $4 \mathrm{~h}$ approximately 54 -fold for IL-6, 1000-fold for IL-8 and 270 -fold for TNF- $\alpha$. Congruently to the experiments during establishment of the model cytokine levels were higher at $4 \mathrm{~h}$ than at $2 \mathrm{~h}$ after incubation. Cytokine concentration time profiles when conventional amphotericin B, liposomal amphotericin B, fluconazole, voriconazole, posaconazole or saline control were added are shown in Fig. 2.

While addition of amphotericin $\mathrm{B}$ further increased the cytokine levels for IL-6, IL- 8 and TNF- $\alpha$ compared to hyphal fragments without drug, this was not the case for the liposomal formulation. Absolute numbers of cytokine concentrations $4 \mathrm{~h}$ after incubation are depicted in Fig. 3a-c for amphotericin. As ratio versus control, conventional amphotericin B increased IL-6, IL-8 and TNF- $\alpha$ : $8.29 \pm 6.33$, $1.12 \pm 0.29$ and $1.37 \pm 0.83$-fold, respectively, while liposomal amphotericin B showed the smallest impact on cytokine release of all five studied antimycotics resulting in $1.08 \pm 0.39,1.01 \pm 0.12$ and $0.93 \pm 0.21$-fold decrease or increase (Fig. 3). cytokine concentrations after incubation of whole blood alone (zero control), with antimycotic agents in whole blood (substance name), with mold alone (AF control), and when antimycotic agents were incubated in combination with mold in whole blood (substance $+\mathrm{AF}$ )

In contrast, the azole antimycotics led to a decrease of cytokine levels for all three studied cytokines and substances-except for the fact that posaconazole did not decrease IL-6 levels (Fig. 3e, f). Fluconazole reduced levels of all three studied cytokines to the greatest extent showing IL-6, IL- 8 and TNF- $\alpha$ ratios of $0.19 \pm 0.17,0.44 \pm 0.23$ and $0.22 \pm 0.14$, respectively (Fig. 3).

Comparing the immunomodulatory effect of antimycotics in blood independent from Aspergillus fumigatus calculating the ratio of cytokine levels at $4 \mathrm{~h}$ for individual antimycotics over zero control without any antimycotic, azoles showed moderate increase or decrease of cytokine levels (Fig. 3). Again, conventional amphotericin B increased cytokine release to a significantly greater extent than the other antimycotic agents resulting to a $61.5 \pm 136$-fold increase of the cytokine levels compared to a $3.2 \pm 4$-fold increase by liposomal amphotericin B (IL-6, IL-8 and TNF- $\alpha$ taken together).

\section{Discussion}

While several studies on the immunomodulatory effect of antibiotics exist [5-9], especially focused on the impact of antifungals on the phagocytic response, the 
immunomodulatory effect of antimycotics has been studied to a definitely smaller extend until now. In the present study, the impact of five antimycotics on cytokine release has been studied systematically in an in vitro aspergillosis model in human whole blood. During establishment of the in vitro aspergillosis model, inactive hyphae induced highest cytokine release whose extent was comparable to previously published LPS models. Studying the impact of antimycotics, the present data show that significant differences in the immunomodulatory potency but also intrinsic potency for cytokine release of available classes of antimycotics do exist. Fluconazole reduced cytokine levels induced by fungal stimulus substantially. However, while liposomal amphotericin B did not impact cytokine release induced by Aspergillus, conventional amphotericin B increased cytokine levels of all three studied cytokines significantly. Knowledge on the immunomodulatory effect of substances might allow using beneficial immunomodulatory effects by co-administration of antimycotics as it is suggested for, e.g., macrolides in case of antibiotics. However, until that is established avoiding harmful immunomodulatory 'side' effects, e.g., by avoiding a significant cytokine increase by, e.g., preferring liposomal amphotericin B to conventional amphotericin B might be of use.

Regarding the establishment of an in vitro aspergillosis model, the inactivated hyphal fragments starting from a conidia count of $10^{6}$ conidia/mL demonstrated the most potent cytokine release when compared to conidia or active hyphal fragments and this was used for the main part of the study. This finding is in accordance to the previous published study by Warris et al. also showing that hyphal fragments induce a greater immunomodulatory response than conidia [16]. Regarding the setup of our experiments, the time points 2 and $4 \mathrm{~h}$ after incubation were chosen congruently with previous LPS experiments and supported by our pilot experiments [20], at time points 2 and $4 \mathrm{~h}$ after incubation. The choice of determining IL 6 and TNF- $\alpha$ allows comparison with results from a previously published study on the immunomodulatory impact of Aspergillus fumigatus [16]. Furthermore, the cytokines IL-6, TNF- $\alpha$ and IL- 8 were identified to be of interest in aspergillosis in previous studies. In general, cytokines are involved in the immune response against fungal pathogens including IL-6, IL-8, IL-10 and TNF- $\alpha$ [25]. High levels of IL-6 and IL-8 were both identified as early predictors of adverse outcomes in invasive pulmonary aspergillosis [26], both being released from epithelial cells of the airways by a transcriptional mechanism [27]. Another study showed that proteases released from Aspergillus fumigatus enhance the production of IL- 6 and IL- 8 by epithelial cells of the airways by a transcriptional mechanism. The authors of this previously published study suggest that downregulating of the cytokines after long exposure to proteases derived by Aspergillus fumigatus may result in a reduced infiltration of $\mathrm{T}$ cells and neutrophils and as consequence lead to the pathogenicity of this fungus [27]. Contrary, TNF- $\alpha$ has been shown to have a protective role in invasive aspergillosis in mice after infection with A. fumigatus [28]. The induction of apoptosis of epithelial cells, induced by TNF- $\alpha$, is suppressed by A. fumigatus leading to the hypothesis that A. fumigatus uses epithelial cells to start dissemination [29]. This might suggest that high TNF- $\alpha$ levels would be beneficial in the defense against invasive aspergillosis. In a previously published study on the cytokine release in aspergillosis and pneumocystis pneumonia, it was shown that IL-6 and IFN-gamma levels were elevated in patients with acute pneumonia, IL-6 being higher in invasive pulmonary aspergillosis [30].

Focusing on the results of the cytokine levels, a 54-fold increase of IL-6 levels from 0 to $4 \mathrm{~h}$ after incubation was seen in our in vitro aspergillosis model. While in the presence of fluconazole cytokine levels solely increased 3.8-fold, conventional amphotericin B led to an cytokine increase of 248 -fold. Looking at the cytokine ratio with/without antifungal agent with 0.19 fluconazole showed the biggest decrease on cytokine reduction and with 8.29 conventional amphotericin B the biggest increase on cytokine levels for IL-6, i.e., the cytokine for which the biggest difference between two antimycotics was found. However, without concomitant stimulation by hyphae fluconazole showed reduction in cytokine release. In this setting, conventional amphotericin B increased cytokine levels significantly compared to liposomal amphotericin $B$ and the other antimycotic agents resulting in a $61.5 \pm 136$-fold increase of cytokine levels compared to $3.2 \pm 4$-fold increase by liposomal amphotericin B.

Obviously inflammatory response is required for fungal clearance, however, dysregulation might damage tissue and harm patients. A previously published, in vitro study investigated the cytokine release induced by Aspergillus fumigatus in whole blood showing a considerable cytokine release, which was higher in blood from patients with chronic granulomatous disease compared to healthy subjects. Therefore, the authors suggest an increased susceptibility to invasive aspergillosis in this patient group [16]. In accordance to the findings of our study, hyphal fragments induced a significantly higher IL-6 and TNF release than conidia did. Another study showed significantly elevated levels of the cytokines IL-6 and IL-8 in serum and of IL-8 in bronchoalveolar lavage fluid in patients with probable/proven invasive pulmonary aspergillosis [31]. Pulmonary aspergillosis, which is associated with poor clinical outcome, may lead to severe hypoxia and as consequence to a severely dysregulation of inflammatory response. One clinical study on targeting inflammatory IL-1 in an aspergillosis model in mice showed a reduction of hypoxia as well as a significant reduction of mortality by IL-1 receptor antagonists [32]. 
Comparing the present results with previously published data on the different classes of antimycotics and first of all focusing on polyene antifungals, a previous murine model studied the capacity of antifungal agents on the TNF- $\alpha$ production by macrophages it was demonstrated that amphotericin B induces TNF- $\alpha$ while fluconazole did not. The authors of the study suggest that this finding would explain the better side effect profile of fluconazole compared to amphotericin B [33]. Another study demonstrated varying immunomodulatory activity of different amphotericin B formulations. Deoxycholate amphotericin B and amphotericin B colloidal dispersion upregulated production of proinflammatory cytokines, while liposomal amphotericin $\mathrm{B}$ and amphotericin B lipid complex downregulated or did not affect proinflammatory cytokine response $[34,35]$. The finding of great variability in the release of proinflammatory cytokines due to divergent amphotericin B formulations is in accordance with the finding of our study comparing conventional and liposomal amphotericin B. One clinical study investigated the infusion-related toxicity of different amphotericin B formulations and its relation to the cytokine release in plasma in neutropenic patients with suspected or documented systemic fungal infection. Liposomal amphotericin $\mathrm{B}$ was better tolerated than conventional amphotericin $\mathrm{B}$ and amphotericin B in lipid emulsion. This formulation also caused the lowest liberation of all three cytokines tested. Though the severity of clinical manifestation could not be correlated with the absolute plasma cytokine levels, still TNF- $\alpha$, IL- 6 and IL-1-RA seemed to play a major role in the acute toxicity of amphotericin B [36]. In the present study, liposomal amphotericin B did solely impact IL-6 levels to $10 \%$ and did not increase TNF- $\alpha$ or IL- 8 levels at all, whereas conventional amphotericin B increased IL-6 8.3-fold.

Regarding azole drugs, a study on the effect of azole antifungals on $\mathrm{T}$ cells demonstrated a variably strongly immunosuppressive effect on human $\mathrm{T}$ lymphocyte proliferation in vitro by azole drugs [37]. In the present study, fluconazole was found to have a substantially greater anti-inflammatory impact than voriconazole and posaconazole. A previously published study comparing TNF- $\alpha$ mRNA expression and production showed a reduced TNF- $\alpha$ production by conventional amphotericin $\mathrm{B}$, which is contrary to the present finding. Voriconazole led to an even smaller TNF- $\alpha$ expression which would again be in accordance to our study. Whether this contrary finding is due to, e.g., the difference in the setting since Choi et al. did not use whole blood but THP 1 cells first treated with drugs for $24 \mathrm{~h}$ before conidia were added, or due to a different reason, remains unknown [19]. In contrast to the significant decrease of TNF- $\alpha$ in human whole blood by fluconazole in the present study, a previous study in a murine candidiasis model showed no impact of fluconazole on TNF- $\alpha$ levels although TNF- $\alpha$ levels in the negative control groups of their and our study were comparable [38]. The reason for this discrepancy is not clear, although species differences might be one explanation. The higher potency of fluconazole compared to the two other azoles may be ascribed to a stronger anti-inflammatory activity, but also by the fact that fluconazole does not harm A. fumigatus while other antimycotics lead to a fungal damage that consequently leads to an anti-inflammatory response. While the used higher concentrations of fluconazole in analogy to clinically observed higher plasma values might be another possible explanation for this finding, the actual reason remains to be investigated. In line with previous studies using LPS as stimulants, standard deviation in general was high and was between 65 and $133 \%$ of the actual mean cytokine levels in the aspergillosis model regardless of the time point, added antimycotics or cytokine. This finding highlights the big intra-individual variability in cytokine release after stimulating blood with either pathogenic additives or antifungals.

For the echinocandin micafungin, it was previously shown to impact the cytokine response to A. fumigatus conidia by increasing IL-10 and decreasing TNF- $\alpha$ in vitro. In a murine model, others were able to show that micafungin induced hyphal damage as well as to modulate the cytokine response leading to an anti-inflammatory response by reducing TNF- $\alpha$ expression and increasing IL-10 expression [18].

Regarding polyene antifungal agents, in a previous murine model study on the capacity of antifungal agents on the TNF- $\alpha$ production by macrophages, it was demonstrated that amphotericin B induces TNF- $\alpha$ while fluconazole did not. The authors of the study suggest that this finding would explain the better side effect profile of fluconazole compared to amphotericin B [33]. Another study demonstrated varying immunomodulatory activity of different amphotericin B formulations. Deoxycholate amphotericin B and amphotericin B colloidal dispersion upregulated production of proinflammatory cytokines, while liposomal amphotericin B and amphotericin B lipid complex downregulated or did not affect proinflammatory cytokine response [34, 35]. The finding of great variability in the release of proinflammatory cytokines due to divergent amphotericin B formulations is in accordance with the finding of our study comparing conventional and liposomal amphotericin B. One clinical study investigated the infusion-related toxicity of different amphotericin B formulations and its relation to the cytokine release in plasma in neutropenic patients with suspected or documented systemic fungal infection. Liposomal amphotericin $\mathrm{B}$ was better tolerated than conventional amphotericin $\mathrm{B}$ and amphotericin B in lipid emulsion. This formulation also caused the lowest liberation of all three cytokines tested. Though the severity of clinical manifestation could not be correlated with the absolute plasma cytokine levels, still TNF- $\alpha$, IL- 6 and IL-1-RA seemed to play a major role 
in the acute toxicity of amphotericin B [36]. In the present study, liposomal amphotericin B did solely impact IL-6 levels to $10 \%$ and did not increase TNF- $\alpha$ or IL- 8 levels at all, whereas conventional amphotericin B increased IL-6 8.3-fold.

Invasive aspergillosis is induced by the inhalation of conidia, which are endocytosed by epithelial cells in the alveoli where conidia germinate to hyphae. Simply put, hyphae penetrate to the abluminal surface of endothelial cells causing cell damage before disseminating hematogenously and then adhering to the luminal endothelial cell surfaces to invade again - once again causing damage [39-41]. This study is limited by the fact that the actual concentration of hyphae disseminating hematogenously is unknown, as well as that solely one A. fumigatus isolate was studied. In addition, endothelial penetration and angioinvasion are followed by a proinflammatory response which is not reflected in this in vitro model. As one further limitation, whole human blood of healthy volunteers was used, not blood from immunocompromised patients. Therefore, cytokine release modeled in this in vitro model using blood from healthy volunteers where endothelial cells are obviously not present might not be identical to the cytokine release in immunocompromised patients. However, an in vitro model never can reflect the complexity of an in vivo setting and the difference between the anti-inflammatory effects of antimycotics still could be pointed out.

Since the applicability of the established aspergillosis model cannot be measured by real-life hyphal concentration for that they are unknown, detected cytokine levels might serve as predictor. Previously presented data from invasive aspergillosis [31] described maximal IL-6 and IL-8 levels in plasma/serum of patients with invasive aspergillosis of $3049.4 \mathrm{pg} / \mathrm{mL}$ and $2300.4 \mathrm{pg} / \mathrm{mL}$, respectively. The IL-6 and IL-8 levels observed in the present study at $4 \mathrm{~h}$ were 35.4 and $5448 \mathrm{pg} / \mathrm{mL}$, thereby reflecting in vivo levels for IL-8 but underestimating levels of IL-6 for blood only stimulated with hyphae. Still, already at these lower IL-6 levels, an enormous increase of cytokines has been shown due to conventional amphotericin $\mathrm{B}$, or a decrease of cytokine release due to fluconazole. We can solely assume that this effect might be even bigger in a real-life scenario in case IL-6 levels in invasive aspergillosis in humans are indeed higher.

In conclusion, this study compared in one in vitro aspergillosis model in whole blood the impact of different antimycotics on the massive cytokine release induced by fungal hyphae of Aspergillus fumigatus. Focusing on antifungal agents, the data of this study indicate significant differences in the immunomodulatory potency of available classes of antimycotics. Fluconazole had the highest anti-inflammatory potency. In contrast, conventional amphotericin B increased cytokine levels induced by Aspergillus fumigatus substantially while liposomal amphotericin B did not impact cytokine release. While this is in accordance to previous studies focusing on the phagocyte response to antifungals, a direct comparison in whole blood has to the best knowledge of the author not been performed until now. This preliminary information might have clinical implication since cytokine dysregulation plays a role in pathogenesis and outcome of fungal infections. However, clinical studies analyzing systematically the impact of different antimycotics on cytokine release as well as a better understanding of the mechanism of immunomodulation of antifungals are warranted to confirm our findings.

Acknowledgements Open access funding provided by Medical University of Vienna. We thank for the financial support obtained by the 'Hochschuljubiläumsfond' of the City of Vienna.

\section{Compliance with ethical standards}

Conflict of interest The authors have no conflict of interest.

Open Access This article is distributed under the terms of the Creative Commons Attribution 4.0 International License (http://creativeco mmons.org/licenses/by/4.0/), which permits unrestricted use, distribution, and reproduction in any medium, provided you give appropriate credit to the original author(s) and the source, provide a link to the Creative Commons license, and indicate if changes were made.

\section{References}

1. Parrillo JE, Parker MM, Natanson C, Suffredini AF, Danner RL, Cunnion RE, et al. Septic shock in humans. Advances in the understanding of pathogenesis, cardiovascular dysfunction, and therapy. Ann Intern Med. 1990;113:227-42.

2. Hack CE, De Groot ER, Felt-Bersma RJ, Nuijens JH, Strack Van Schijndel RJ, Eerenberg-Belmer AJ, et al. Increased plasma levels of interleukin-6 in sepsis. Blood. 1989;74:1704-10.

3. Giamarellos-Bourboulis EJ. Immunomodulatory therapies for sepsis: unexpected effects with macrolides. Int J Antimicrob Agents. 2008;32:39-43.

4. Eichacker PQ, Parent C, Kalil A, Esposito C, Cui X, Banks SM, et al. Risk and the efficacy of antiinflammatory agents: retrospective and confirmatory studies of sepsis. Am J Respir Crit Care Med. 2002;166:1197-205.

5. Ianaro A, Ialenti A, Maffia P, Sautebin L, Rombolà L, Carnuccio R, et al. Anti-inflammatory activity of macrolide antibiotics. J Pharmacol Exp Ther. 2000;292:156-63.

6. Lotter K, Hocherl K, Bucher M, Kees F. In vivo efficacy of telithromycin on cytokine and nitric oxide formation in lipopolysaccharide-induced acute systemic inflammation in mice. J Antimicrob Chemother. 2006;58:615-21.

7. Tauber SC, Nau R. Immunomodulatory properties of antibiotics. Curr Mol Pharmacol. 2008;1:68-79.

8. Giuliani F, Hader W, Yong VW. Minocycline attenuates T cell and microglia activity to impair cytokine production in T cellmicroglia interaction. J Leukoc Biol. 2005;78:135-43.

9. Dalhoff A. Immunomodulatory activities of fluoroquinolones. Infection. 2005;33:55-70.

10. Dalhoff A, Shalit I. Immunomodulatory effects of quinolones. Lancet Infect Dis. 2003;3:359-71. 
11. Martin-Loeches I, Lisboa T, Rodriguez A, Putensen C, Annane D, Garnacho-Montero J, et al. Combination antibiotic therapy with macrolides improves survival in intubated patients with community-acquired pneumonia. Intensive Care Med. 2010;36:612-20.

12. Restrepo MI, Mortensen EM, Waterer GW, Wunderink RG, Coalson JJ, Anzueto A. Impact of macrolide therapy on mortality for patients with severe sepsis due to pneumonia. Eur Respir J. 2009;33:153-9.

13. Cornely OA, Aversa F, Cook P, Jones B, Michallet M, Shea T, et al. Evaluating the role of prophylaxis in the management of invasive fungal infections in patients with hematologic malignancy. Eur J Haematol. 2011;87:289-301.

14. Lass-Florl C. The changing face of epidemiology of invasive fungal disease in Europe. Mycoses. 2009;52:197-205.

15. Lin SJ, Schranz J, Teutsch SM. Aspergillosis case-fatality rate: systematic review of the literature. Clin Infect Dis. 2001;32:358-66.

16. Warris A, Netea MG, Wang JE, Gaustad P, Kullberg BJ, Verweij $\mathrm{PE}$, et al. Cytokine release in healthy donors and patients with chronic granulomatous disease upon stimulation with Aspergillus fumigatus. Scand J Infect Dis. 2003;35:482-7.

17. Romani L. Immunity to fungal infections. Nat Rev Immunol. 2011;11:275-88.

18. Moretti S, Bozza S, Massi-Benedetti C, Prezioso L, Rossetti E, Romani L, et al. An immunomodulatory activity of micafungin in preclinical aspergillosis. J Antimicrob Chemother. 2014;69:1065-74.

19. Choi JH, Kwon EY, Park CM, Choi SM, Lee DG, Yoo JH, et al. Immunomodulatory effects of antifungal agents on the response of human monocytic cells to Aspergillus fumigatus conidia. Med Mycol. 2010;48:704-9.

20. Zeitlinger M, Marsik C, Steiner I, Sauermann R, Seir K, Jilma B, et al. Immunomodulatory effects of fosfomycin in an endotoxin model in human blood. J Antimicrob Chemother. 2007;59:219-23.

21. B.V. TP. Summary of Product Characteristics 2017. https://www. medicines.org.uk/emc/product/5407.

22. Ltd CI-fFP. Summary of Product Characteristics Fluconazole $2 \mathrm{mg} / \mathrm{ml}$ Solution for Infusion 2017. https://www.medicines.org. uk/emc/product/3378.

23. Limited APE. Summary of Product Characteristics Voriconazole $200 \mathrm{mg}$ powder for solution for infusion 2016. https://www.medic ines.org.uk/emc/product/8674.

24. Courtney R, Pai S, Laughlin M, Lim J, Batra V. Pharmacokinetics, safety, and tolerability of oral posaconazole administered in single and multiple doses in healthy adults. Antimicrob Agents Chemother. 2003;47:2788-95.

25. Antachopoulos C, Roilides E. Cytokines and fungal infections. Br J Haematol. 2005;129:583-96.

26. Vonk AG, Earnest A, Kullberg B-J, Schlamm HT, Chai LYA, Netea MG, et al. Early proinflammatory cytokines and C-reactive protein trends as predictors of outcome in invasive Aspergillosis. J Infect Dis. 2010;202:1454-62.

27. Borger P, Koeter GH, Timmerman JA, Vellenga E, Tomee JF, Kauffman HF. Proteases from Aspergillus fumigatus induce interleukin (IL)-6 and IL-8 production in airway epithelial cell lines by transcriptional mechanisms. J Infect Dis. 1999;180:1267-74.
28. Nagai H, Guo J, Choi H, Kurup V. Interferon-gamma and tumor necrosis factor-alpha protect mice from invasive aspergillosis. J Infect Dis. 1995; 172:1554-60.

29. Balloy V, Sallenave J-M, Wu Y, Touqui L, Latgé J-P, Si-Tahar $\mathrm{M}$, et al. Aspergillus fumigatus-induced interleukin-8 synthesis by respiratory epithelial cells is controlled by the phosphatidylinositol 3-kinase, p38 MAPK, and ERK1/2 pathways and not by the toll-like receptor-MyD88 pathway. J Biol Chem. 2008;283:30513-21.

30. Shen HP, Tang YM, Song H, Xu WQ, Yang SL, Xu XJ. Efficiency of interleukin 6 and interferon gamma in the differentiation of invasive pulmonary aspergillosis and pneumocystis pneumonia in pediatric oncology patients. Int J Infect Dis. 2016;48:73-7.

31. Heldt S, Prattes J, Eigl S, Spiess B, Flick H, Rabensteiner J, et al. Diagnosis of invasive aspergillosis in hematological malignancy patients: performance of cytokines, Asp LFD, and Aspergillus PCR in same day blood and bronchoalveolar lavage samples. J Infect. 2018;77:235-41.

32. Gresnigt MS, Rekiki A, Rasid O, Savers A, Jouvion G, Dannaoui E, et al. Reducing hypoxia and inflammation during invasive pulmonary aspergillosis by targeting the interleukin- 1 receptor. Sci Rep. 2016;6:26490.

33. Louie A, Baltch AL, Franke MA, Smith RP, Gordon MA. Comparative capacity of four antifungal agents to stimulate murine macrophages to produce tumour necrosis factor alpha: an effect that is attenuated by pentoxifylline, liposomal vesicles, and dexamethasone. J Antimicrob Chemother. 1994;34:975-87.

34. Simitsopoulou M, Roilides E, Dotis J, Dalakiouridou M, Dudkova F, Andreadou E, et al. Differential expression of cytokines and chemokines in human monocytes induced by lipid formulations of amphotericin B. Antimicrob Agents Chemother. 2005;49:1397-403.

35. Simitsopoulou M, Roilides E, Walsh TJ. Immunomodulatory properties of antifungal agents on phagocytic cells. Immunol Invest. 2011;40:809-24.

36. Arning M, Kliche KO, Heer-Sonderhoff AH, Wehmeier A. Infusion-related toxicity of three different amphotericin B formulations and its relation to cytokine plasma levels. Mycoses. 1995;38:459-65.

37. Pawelec G, Ehninger G, Rehbein A, Schaudt K, Jaschonek $\mathrm{K}$. Comparison of the immunosuppressive activities of the antimycotic agents itraconazole, fluconazole, ketoconazole and miconazole on human T-cells. Int J Immunopharmacol. 1991;13:299-304.

38. Kalkanci A, Kustimur S. The effect of fluconazole treatment on tumor necrosis factor-alpha production in murine candidiasis. Yale J Biol Med. 2002;75:241-5.

39. Filler SG, Sheppard DC. Fungal invasion of normally non-phagocytic host cells. PLoS Pathog. 2006;2:e129.

40. Ben-Ami R, Lewis RE, Kontoyiannis DP. Enemy of the (immunosuppressed) state: an update on the pathogenesis of Aspergillus fumigatus infection. Br J Haematol. 2010;150:406-17.

41. Chotirmall SH, Al-Alawi M, Mirkovic B, Lavelle G, Logan PM, Greene CM, et al. Aspergillus-associated airway disease, inflammation, and the innate immune response. Biomed Res Int. 2013;2013:723129. 